The relationship between phase transitions and percolation in models with ground-state degeneracy

To cite this article: Azer Kerimov 2002 J. Phys. A: Math. Gen. 355365

View the article online for updates and enhancements.
Related content

$$
\begin{aligned}
& \text { - } \frac{\text { Countable extreme Gibbs states in a one- }}{\frac{\text { dimensional model with unique ground }}{\text { Azate }}} \\
& \text { - A berimov } \\
& \frac{\text { dimensional ferromagnetical Ising model }}{\text { Azer Kerimov }} \\
& -\frac{\text { A disagreement-percolation type }}{\text { uniqueness condition for Gibbs states in }} \\
& \frac{\text { models with long-range interactions }}{\text { Azer Kerimov }}
\end{aligned}
$$

Recent citations

\begin{tabular}{l} 
- $\frac{\text { A disagreement-percolation type }}{\text { uniqueness condition for Gibbs states in }}$ \\
$\frac{\text { models with long-range interactions }}{\text { Azer Kerimov }}$ \\
- ONE-DIMENSIONAL LONG RANGE \\
$\frac{\text { WIDOM-ROWLINSON MODEL WITH }}{\text { PERIODIC PARTICLE ACTIVITIES }}$ \\
\hline AHMET SENSOY \\
- GROUND STATES OF ONE- \\
$\frac{\text { DIMENSIONAL LONG-RANGE }}{\text { FERROMAGNETIC ISING MODEL WITH }}$ \\
$\frac{\text { EXTERNAL FIELD }}{\text { AZER KERIMOV }}$
\end{tabular} 


\title{
The relationship between phase transitions and percolation in models with ground-state degeneracy
}

\author{
Azer Kerimov \\ Department of Mathematics, Bilkent University, 06533 Bilkent, Ankara, Turkey \\ E-mail: kerimov@fen.bilkent.edu.tr
}

Received 1 October 2001, in final form 21 May 2002

Published 21 June 2002

Online at stacks.iop.org/JPhysA/35/5365

\begin{abstract}
A criterion for the uniqueness of limiting Gibbs states in classical models with unique ground states is formulated. Various applications of this criterion formulated in the terminology of percolation theory are discussed.
\end{abstract}

PACS numbers: 64.60.Ak, 05.50.+q

\section{Introduction and the formulation of results}

The problem of the absence of phase transitions is one of the most central problems in statistical physics. The investigation of this problem is based on several different approaches.

The most powerful tool is the method of cluster expansions. In cluster expansions theory local characteristics of a field (finite-dimensional distributions, mean values of local functions, etc) are represented in terms of a series where each term depends on a finite group of field variables (so-called clusters) and are expressed in terms of these characteristics in an explicit way. Unfortunately, however, modern cluster expansion theory is only applicable when the model has a small parameter, in other words, the method appears as a version of the perturbation theory. Many limiting Gibbs states uniqueness theorems are obtained by this method in different situations: in the low-temperature ferromagnetic region, in the region of large magnetization, in the high temperature region, etc. The origins of the notion of a cluster playing a central role in all of these results goes back to a pioneering paper by Peierls [1]. Rigorous mathematical theory of cluster expansions was developed in a series of papers as [2-13].

The other known theory is the method of estimation of the total interaction of a given spin with all other spins, which is based on a kind of contraction argument. This method is due to Dobrushin [14].

There is also the uniqueness condition, which is only applicable to one-dimensional models. This condition states that the interaction between very distant spins should decrease so rapidly that the total interaction energy of the spins on any two complementary half-lines 
is finite [14-17]. The result of [17, 18] is a successful application of the methods of [14] in one dimension. For example, in the model satisfying the condition $\sum_{r \in \mathbf{Z}^{1}, r>0} r|U(r)|<\infty$ $(U(r)$ is a pair potential of long range) the limit of the Gibbs states is unique.

In this paper we give an alternate method for establishing the absence of phase transition. This method reduces the problem of uniqueness of limiting Gibbs states to a problem of percolation of special clusters. On one hand the method only works in models with a unique ground state, while on the other hand the method allows us to establish uniqueness for longrange potentials. In two- or higher-dimensional models most classical results are obtained for finite-range potentials [3-5, 8, 9], but our method allows one to obtain uniqueness theorems without complicated cluster expansions in models with long-range interactions. The method is especially powerful in one-dimensional models with very slowly decreasing potentials (see the example in section 3, the classical methods mentioned above fail in this case). The origin of the main idea of this method comes from [18], where the uniqueness theorem for the one-dimensional antiferromagnetical model was established. In [19] a very sophisticated zero-temperature phase diagram of this model was investigated and the hypothesis on the uniqueness of limiting Gibbs states was formulated (since the potential of this model does not satisfy the strong decreasing conditions of [14-17] the classical methods fail to prove uniqueness).

Consider a model on $\mathbf{Z}^{v}$ with the formal Hamiltonian

$$
H(\phi)=\sum_{B \subset \mathbf{Z}^{v}} U(\phi(B))
$$

where the spin variables $\phi(x) \in \Phi$, $\Phi$ is a finite set, $\phi(B)$ denotes the restriction of the configuration $\phi$ to the set $B$ and the potential $U(\phi(B))$ is a translationally invariant function.

We impose a natural condition on the potential $U(\phi(B))$, necessary for the existence of the thermodynamic limit:

$$
\sum_{B \subset \mathbf{Z}^{v}: x \in B}|U(\phi(B))|<\text { constant }
$$

where the constant does not depend on the configuration of $\phi$.

A configuration $\phi^{g r}$ is said to be a ground state, if the expression $H\left(\phi^{\prime}\right)-H\left(\phi^{g r}\right)$ is non-negative for any finite perturbation $\phi^{\prime}$ of $\phi^{g r}$. We assume that model (1) has a unique ground state. The main idea of the method is as follows.

Let $V_{N}$ be a $v$-cube with the centre at the origin and with an edge length of $2 N: V_{N}=\left\{x_{1}, x_{2}, \ldots, x_{v}:-N \leqslant x_{i} \leqslant N, i=1,2, \ldots, v\right\}$. We denote the set of all configurations $\phi\left(V_{N}\right)$ by $\Phi(N)$. Suppose that the boundary conditions $\phi^{i}, i=1,2$ are fixed. Let $\mathbf{P}_{N}^{i}$ be the Gibbs distribution on $\Phi(N)$ corresponding to the boundary conditions $\phi^{i}, i=1,2$. Take $M<N$ and let $\mathbf{P}_{N}^{i}\left(\phi^{\prime}\left(V_{M}\right)\right)$ be the probability of the event that the restriction of the configuration $\phi\left(V_{N}\right)$ to $V_{M}$ coincides with $\phi^{\prime}\left(V_{M}\right)$.

The concatenation of the configurations $\phi\left(V_{N}\right)$ and $\phi^{i}\left(\mathbf{Z}^{v}-V_{N}\right)$ is denoted by $\chi: \chi(x)=\phi(x)$, if $x \in V_{N}$ and $\chi(x)=\phi^{i}(x)$, if $x \in \mathbf{Z}^{v}-V_{N}$. Define

$$
H_{N}\left(\phi \mid \phi^{i}\right)=\sum_{B \subset \mathbf{Z}^{\nu}: B \cap V_{N} \neq \emptyset} U(\chi(B)) .
$$

Let $\Phi^{\min }\left(N, \phi^{i}\right)$ be the set of configurations with minimal energy at fixed $N$ and boundary conditions $\phi^{i}$, and $\phi_{N}^{\min , i}$ be an arbitrarily chosen representative of this set:

$$
H_{N}\left(\phi_{N}^{\min , i} \mid \phi^{i}\right)=\min _{\phi \in \Phi(N)} H_{N}\left(\phi \mid \phi^{i}\right)
$$


In the following $H_{N}\left(\phi \mid \phi^{i}, \phi_{N}^{\min , i}\right)$ denotes the relative energy of a configuration $\phi$ (with respect to $\left.\phi_{N}^{\min , i}\right)$ :

$$
H_{N}\left(\phi \mid \phi^{i}, \phi_{N}^{\min , i}\right)=H_{N}\left(\phi \mid \phi^{i}\right)-H_{N}\left(\phi_{N}^{\min , i} \mid \phi^{i}\right) .
$$

Since the ground state of model (1) is unique, the configuration $\phi_{N}^{\min , i}$ almost coincides with the ground state $\varphi^{g r}$ (see lemma 1 in section 2). Let $\mathbf{P}_{N}^{i}$ be the Gibbs distributions on $\Phi(N)$ corresponding to the boundary conditions $\phi^{i}, i=1,2$ defined using the relative energies of configurations. Take $M<N$ and let $\mathbf{P}_{N}^{i}\left(\phi^{\prime}\left(V_{M}\right)\right)$ be the probability of the event that the restriction of the configuration $\phi\left(V_{N}\right)$ to $V_{M}$ coincides with $\phi^{\prime}\left(V_{M}\right)$.

Let $\mathbf{P}^{1}$ and $\mathbf{P}^{2}$ be two extreme limiting Gibbs states of the model (1). Since two extreme limiting Gibbs states are singular or coincide, we can prove the uniqueness of the limiting Gibbs states of model (1) by showing that $\mathbf{P}^{1}$ and $\mathbf{P}^{2}$ are not singular: two positive constants constant $_{1}$ and constant 2 exist, for any $M$ and $\phi^{\prime}\left(V_{M}\right)$ a number $N_{0}(M)$ exists such that for any $N>N_{0}$

$$
\text { constant }_{1}<\mathbf{P}_{N}^{1}\left(\phi^{\prime}\left(V_{M}\right)\right) / \mathbf{P}_{N}^{2}\left(\phi^{\prime}\left(V_{M}\right)\right)<\text { constant }_{2} .
$$

The first important point is the introduction of the contour model common for boundary conditions $\phi^{i}, i=1,2$ (a contour is a connected subconfiguration not coinciding with the ground state). After that, by using a well known trick [20] we obtain 'non-interacting' clusters from interacting contours (a cluster is a collection of contours connected by interaction bonds).

The second important point is the combinatorial lemma 3, which allows us to reduce the dependence of the expression $\mathbf{P}_{N}^{1}\left(\phi\left(V_{M}\right)\right) / \mathbf{P}_{N}^{2}\left(\phi\left(V_{M}\right)\right)$ on the boundary conditions $\phi^{1}$ and $\phi^{2}$ to the sum of the statistical weights of the 2-clusters connecting the cube $V_{M}$ with the boundary (so-called long 2-clusters). Since the statistical weight of the 2-cluster is not necessarily positive, we estimate the sum of the absolute values of the statistical weights of the long 2-clusters. Thus, we reduce the uniqueness of the limiting Gibbs states problem to the percolation-like problem of the estimation of the sum of some clusters connecting $V_{M}$ with the boundary.

The criterion formulated works at all dimensions, and for models with a very long-range interaction. Since in low dimensions percolation is the more rarely observed phenomenon, the criterion is especially powerful in the one-dimensional case. The decreasing conditions imposed on the potential in uniqueness theorem 4 are more general; the results of [14-18] are obtained under stronger decreasing conditions on the potential.

In many cases the uniqueness problem after the reduction to a percolation type problem has a rather natural and simple solution.

We now start to formulate the main criterion of uniqueness. Suppose that the boundary conditions $\phi^{1}$ are fixed. Consider the $\mathbf{P}_{N}^{1}$ probability of the event that the restriction of the configuration $\phi\left(V_{N}\right)$ to $V_{M}$ coincides with $\phi^{\prime}\left(V_{M}\right)$ :

$$
\begin{aligned}
& \mathbf{P}_{N}^{1}\left(\phi^{\prime}\left(V_{M}\right)\right)=\frac{\sum_{\phi\left(V_{N}\right): \phi\left(V_{M}\right)=\phi^{\prime}\left(V_{M}\right)} \exp \left(-\beta H_{N}\left(\phi\left(V_{N}\right) \mid \phi^{1}, \phi_{N}^{\mathrm{min}, 1}\right)\right)}{\sum_{\phi\left(V_{N}\right)} \exp \left(-\beta H_{N}\left(\phi\left(V_{M}\right) \mid \phi^{1}, \phi_{N}^{\min , 1}\right)\right)} \\
& =\frac{\exp \left(-\beta H_{M}^{\mathrm{in}}\left(\phi^{\prime}\left(V_{M}\right)\right) Y\left(\phi^{\prime}\left(V_{M}\right), V_{N}, \phi^{1}\right) \Xi\left(V_{N}-V_{M} \mid \phi^{1}, \phi^{\prime}\left(V_{M}\right), \phi_{N}^{\min , 1}\right)\right.}{\sum_{\phi^{\prime \prime}\left(V_{M}\right)} \exp \left(-\beta H_{M}^{\mathrm{in}}\left(\phi^{\prime \prime}\left(V_{M}\right)\right)\right) Y\left(\phi^{\prime \prime}\left(V_{M}\right), V_{N}, \phi^{1}\right) \Xi\left(V_{N}-V_{M} \mid \phi^{1}, \phi^{\prime \prime}\left(V_{M}\right), \phi_{N}^{\min , 1}\right)} \\
& =\frac{\exp \left(-\beta H_{M}^{\mathrm{in}}\left(\phi^{\prime}\left(V_{M}\right)\right) Y\left(\phi^{\prime}\left(V_{M}\right), V_{N}, \phi^{1}\right) \Xi^{\phi^{1}, \phi^{\prime}}\right.}{\sum_{\phi^{\prime \prime}\left(V_{M}\right)} \exp \left(-\beta H_{M}^{\mathrm{in}}\left(\phi^{\prime \prime}\left(V_{M}\right)\right) Y\left(\phi^{\prime \prime}\left(V_{M}\right), V_{N}, \phi^{1}\right) \Xi^{\phi^{1}, \phi^{\prime \prime}}\right.}
\end{aligned}
$$


where the summation in $\sum_{\phi^{\prime \prime}\left(V_{M}\right)}$ is taken over all the possible configurations of $\phi^{\prime \prime}\left(V_{M}\right)$, $H_{M}^{\mathrm{in}}\left(\phi^{\prime}\left(V_{M}\right)\right)=\sum_{B \subset V_{M}} U\left(\phi^{\prime}(B)\right)-U\left(\phi_{N}^{\mathrm{min}, 1}\right)$ and $H_{M}^{\mathrm{in}}\left(\phi^{\prime \prime}\left(V_{M}\right)\right)=\sum_{B \subset V_{M}} U\left(\phi^{\prime \prime}(B)\right)-$ $U\left(\phi_{N}^{\min , 1}\right)$ are the interior relative energies of $\phi^{\prime}\left(V_{M}\right)$ and $\phi^{\prime \prime}\left(V_{M}\right) . \Xi^{\phi^{1}, \phi^{\prime}}$ and $\Xi^{\phi^{1}, \phi^{\prime \prime}}$ denote the partition functions corresponding to the boundary conditions $\phi^{1}\left(\mathbf{Z}^{v}-V_{N}\right), \phi^{\prime}\left(V_{M}\right), \phi^{\prime \prime}\left(V_{M}\right)$ :

$$
\begin{aligned}
& \Xi^{\phi^{1}, \phi^{\prime}}=\Xi\left(V_{N}-V_{M} \mid \phi^{1}, \phi^{\prime}\left(V_{M}\right), \phi_{N}^{\min , 1}\right) \\
& \Xi^{\phi^{1}, \phi^{\prime \prime}}=\Xi\left(V_{N}-V_{M} \mid \phi^{1}, \phi^{\prime \prime}\left(V_{M}\right), \phi_{N}^{\min , 1}\right) .
\end{aligned}
$$

The factor $Y\left(\phi\left(V_{M}\right), V_{N}, \phi^{1}\right)$ is defined as

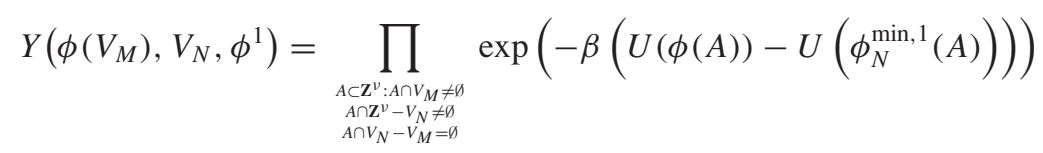

where $\phi$ in (5) is equal to $\phi^{\prime}$ for $x \in V_{M}$ and is equal to $\phi^{1}$ for $x \in \mathbf{Z}^{v}-V_{N}$.

Expression (5) gives the 'direct' interaction of $\phi\left(V_{M}\right)$ with the boundary conditions $\phi^{1}\left(\mathbf{Z}^{v}-V_{N}\right)$.

Let us consider the partition functions $\Xi^{\phi^{1}, \phi^{\prime \prime}}=\Xi\left(V_{N}-V_{M} \mid \phi^{1}, \phi^{\prime \prime}\left(V_{M}\right), \phi_{N}^{\min , 1}\right)$ corresponding to the boundary conditions $\phi^{1}\left(\mathbf{Z}^{\nu}-V_{N}\right), \phi^{\prime \prime}\left(V_{M}\right)$ and $\Xi^{\phi^{2}, \phi^{\prime}}=\Xi\left(V_{N}-V_{M} \mid \phi^{2}\right.$, $\left.\phi^{\prime}\left(V_{M}\right), \phi_{N}^{\min , 2}\right)$ corresponding to the boundary conditions $\phi^{2}\left(\mathbf{Z}^{\nu}-V_{N}\right), \phi^{\prime}\left(V_{M}\right)$ as in (4).

Define a super-partition function

$$
\begin{aligned}
\left(\Xi^{\phi^{1}, \phi^{\prime \prime}} \Xi^{\phi^{2}, \phi^{\prime}}\right) & =\sum \exp \left(-\beta H_{N}\left(\phi^{3}\left(V_{N}\right) \mid \phi^{1}, \phi^{\prime \prime}, \phi_{V}^{\min , 1}\right)\right) \\
& \times \exp \left(-\beta H_{N}\left(\phi^{4}\left(V_{N}\right) \mid \phi^{2}, \phi^{\prime}, \phi_{N}^{\min , 2}\right)\right)
\end{aligned}
$$

where the summation is taken over all the pairs of configurations $\phi^{3}\left(V_{N}\right)$ and $\phi^{4}\left(V_{N}\right)$, such that $\phi^{3}\left(V_{M}\right)=\phi^{\prime \prime}\left(V_{M}\right), \phi^{4}\left(V_{M}\right)=\phi^{\prime}\left(V_{M}\right)$.

Consider the partition of $\mathbf{Z}^{v}$ into $v$-cubes $V_{R}(x)$, where $V_{R}(x)$ is a cube with an edge length of $R$ and with its centre at $x=\left(x_{1}, \ldots, x_{v}\right)$, where $x_{i}=R / 2+k_{i} R ; i=1,2, \ldots, v ; k_{i}$ is an integer number.

Let us consider an arbitrary configuration $\phi$. We say that a cube $V_{R}(x)$ is not regular, if $\phi\left(V_{R}(x)\right) \neq \phi^{g r}\left(V_{R}(x)\right)$. Two non-regular cubes are called connected provided that their intersection is not empty. The connected components of non-regular segments defined in this way are called supports of contours and are denoted by supp $K$.

A pair $K=(\operatorname{supp} K, \phi(\operatorname{supp} K))$ is called a contour.

Obviously, for each contour $K$, a configuration $\psi_{K}$ exists such that the only contour of the configuration $\psi_{K}$ is $K\left(\psi_{K}\right.$ on $\mathbf{Z}^{v}-\operatorname{supp} K$ coincides with $\phi^{g r}$ ).

The weight of contour $K$ can be calculated using the following formula:

$$
\gamma(K)=H\left(\psi_{K}\right)-H\left(\phi^{g r}\right) .
$$

The statistical weight of a contour is

$$
w\left(K_{i}\right)=\exp \left(-\beta \gamma\left(K_{i}\right)\right) .
$$

The following equation is a direct consequence of the formulae (6) and (7)

$$
\exp \left(-\beta H_{N}\left(\phi \mid \phi^{1}, \phi_{N}^{\min , 1}\right)=\prod_{i=1}^{n} w\left(K_{i}\right) \exp \left(-\beta G\left(K_{1}, \ldots, K_{n}\right)\right)\right.
$$


where the multiplier $G\left(K_{1}, \ldots, K_{n}\right)$ corresponds to the interaction between the contours and the boundary conditions $\phi^{1}$.

$$
G\left(K_{1}, \ldots, K_{n}\right)=\sum_{k=2}^{n} \sum_{i_{1}, \ldots, i_{k}} G\left(K_{i_{1}}, \ldots, K_{i_{k}}\right)
$$

where at each fixed $k$ the summation is taken over all possible non-ordered collections $i_{1}, \ldots, i_{k}$.

The interaction between $K_{i_{1}}, \ldots, K_{i_{k}}$ arises due to the fact that the weight of the contour $K_{i_{j}}, j=1, \ldots, k$ was calculated under the assumption that the configuration outside $\operatorname{supp}\left(K_{i_{j}}\right)$ coincides with the ground state.

The set of all interaction terms in the double sum (9) will be denoted by $I G$. We now write (8) as follows:

$$
\begin{gathered}
\exp \left(-\beta H_{N}\left(\phi \mid \phi^{1}, \phi_{N}^{\min , 1}\right)\right)=\prod_{i=1}^{n} w\left(K_{i}\right) \prod_{B \in I G}\left(\exp \left(-\beta G\left(K_{i_{1}}, \ldots, K_{i_{k}}\right)\right)\right) \\
=\prod_{i=1}^{n} w\left(K_{i}\right) \prod_{G \in I G}\left(1+\exp \left(-\beta G\left(K_{i_{1}}, \ldots, K_{i_{k}}\right)-1\right)\right) .
\end{gathered}
$$

From (10) we obtain

$$
\exp \left(-\beta H\left(\phi \mid \phi^{1}, \phi_{N}^{\min , 1}\right)\right)=\sum_{I G^{\prime} \subset I G} \prod_{i \in \mathbf{I}} w\left(K_{i}\right) \prod_{G \in I G^{\prime}} g(G)
$$

where the summation is taken over all subsets $I G^{\prime}$ (including the empty set) of the set $I G$, and $g(G)=\exp (-\beta G)-1$.

Consider an arbitrary term of the sum (11), which corresponds to the subset $I G^{\prime} \subset I G$. Let the interaction element $G \in I G^{\prime}$. Consider the set $\mathbf{K}$ of all contours such that for each contour $K \subset \mathbf{K}$, the set supp $K \cap G$ is non-empty. We call any two contours from $\mathbf{K}$ neighbours in $I G^{\prime}$ interaction. The set of contours $K^{\prime}$ is called connected in the $I G^{\prime}$ interaction if for any two contours $K_{p}$ and $K_{q}$ a collection $\left(K_{1}=K_{p}, K_{2}, \ldots, K_{n}=K_{q}\right)$ exists such that any two contours $K_{i}$ and $K_{i+1}, i=1, \ldots, n-1$, are neighbours.

The pair $D=\left[\left(K_{i}, i=1, \ldots, s\right) ; I G^{\prime}\right]$, where $I G^{\prime}$ is a set of interaction elements, is called a cluster provided a configuration $\phi$ containing all $K_{i} ; i=1, \ldots, s ; I G^{\prime} \subset I G$ exists; and the set $\left(K_{i}, i=1, \ldots, s\right)$ is connected in $I G^{\prime}$ interaction. The statistical weight of a cluster $D$ is defined using the formula

$$
w(D)=\prod_{i=1}^{s} w\left(K_{i}\right) \prod_{(x, y) \in I G^{\prime}} g(G) .
$$

Note that $w(D)$ is not necessarily positive.

Two clusters $D_{1}$ and $D_{2}$ are called compatible provided any two contours $K_{1}$ and $K_{2}$ belonging to $D_{1}$ and $D_{2}$, respectively, are compatible. A set of clusters is called compatible provided any two clusters of it are compatible.

If $D=\left[\left(K_{i}, i=1, \ldots, s\right) ; I G^{\prime}\right]$, then we say that $K_{i} \in D ; i=1, \ldots, s$.

If $\left[D_{1}, \ldots, D_{m}\right]$ is a compatible set of clusters and $\bigcup_{i=1}^{m} \operatorname{supp} D_{i} \subset V_{N}$, then a configuration $\phi$ which contains this set of clusters exists. For each configuration $\phi$ we have

$$
\exp \left(-\beta H_{N}\left(\phi \mid \phi^{1}, \phi_{N}^{\min , 1}\right)\right)=\sum_{I G^{\prime} \subset I G} \prod w\left(D_{i}\right)
$$


where the clusters $D_{i}$ are completely determined by the set $I G^{\prime}$. The partition function is

$$
\Xi\left(\phi^{1}\right)=\sum w\left(D_{1}\right) \cdots w\left(D_{m}\right)
$$

where the summation is taken over all non-ordered compatible collections of clusters.

Thus, we obtain non-interacting clusters from interacting contours [20].

The following generalization of the definition of compatibility allows us to represent $\left(\Xi^{\phi^{1}, \phi^{\prime \prime}} \Xi^{2, \prime}\right)$ as a single-partition function.

A set of clusters is called 2-compatible provided any of its two parts coming from two Hamiltonians are compatible. In other words, in 2-compatibility an intersection of supports of two clusters coming from different partition functions is allowed.

If $\left[D_{1}, \ldots, D_{m}\right]$ is a 2-compatible set of clusters and $\bigcup_{i=1}^{m} \operatorname{supp} D_{i} \subset V_{N}-V_{M}$, then two configurations $\phi^{3}$ and $\phi^{4}$ which contain this set of clusters exist. For each pair of configurations $\phi^{3}$ and $\phi^{4}$ we have

$\exp \left(-\beta H_{N}\left(\phi^{3} \mid \phi^{1}, \phi_{N}^{\min , 1}\right) \exp \left(-\beta H_{N}\left(\phi^{4} \mid \phi^{2}, \phi_{N}^{\min , 2}\right)=\sum_{I G^{\prime} \subset I G, I G^{\prime \prime} \subset I G} \prod w\left(D_{i}\right)\right.\right.$

where the clusters $D_{i}$ are completely determined by the sets $I G^{\prime}$ and $I G^{\prime \prime}$.

The double-partition function is

$$
\Xi^{\phi^{1}, \phi^{\prime \prime}, \phi^{2}, \phi^{\prime}}=\Xi^{\phi^{1}, \phi^{\prime \prime}} \Xi^{\phi^{2}, \phi^{\prime}}=\sum w\left(D_{1}\right) \cdots w\left(D_{m}\right)
$$

where the summation is taken over all non-ordered 2-compatible collections of clusters.

Let $w\left(D_{1}\right) \cdots w\left(D_{m}\right)$ be a term of the double-partition function $\Xi^{\phi^{1}, \phi^{\prime \prime}, \phi^{2}, \phi^{\prime}}$. The connected components of the collection $\left[\operatorname{supp}\left(D_{1}\right), \ldots, \operatorname{supp}\left(D_{m}\right)\right]$ are the supports of the general clusters. A general cluster $S D$ is a pair $(\operatorname{supp}(S D), \phi(\operatorname{supp}(S D))$. In the following, instead of the expression 'generally compatible collection of clusters' we use the expression 'compatible collection of 2-clusters'.

A 2-cluster $S D=\left[\left(D_{i}, i=1, \ldots, m\right) ; I G^{\prime}, I G^{\prime \prime}\right]$ is said to be long if the intersection of the set $\left(\bigcup_{i=1}^{m} \operatorname{supp} D_{i}\right) \cup I G^{\prime} \cup I G^{\prime \prime}$ with both $V_{M}$ and $\mathbf{Z}^{v}-V_{N}$ is non-empty. In other words, a long 2-cluster connects the boundary with the cube $V_{M}$ by using its contours and bonds.

A set of 2-clusters is called compatible provided the set of all clusters belonging to these 2-clusters are 2-compatible.

Definition 1. We say that model (1) has a not-long 2-clusters property, if a number $\epsilon, 0<\epsilon<1$ exists such that for each fixed cube $V_{M}$, a number $N_{0}=N_{0}(M)$, which depends on $M$ only exists such that if $N>N_{0}$, then

$(1-\epsilon) \Xi^{\phi^{1}, \phi^{\prime}, \phi^{2}, \phi^{\prime \prime}}<\Xi^{\phi^{1}, \phi^{\prime}, \phi^{2}, \phi^{\prime \prime},(n . l .)}=\sum w\left(S D_{1}\right) \cdots w\left(S D_{m}\right)<(1+\epsilon) \quad \Xi^{\phi^{1}, \phi^{\prime}, \phi^{2}, \phi^{\prime \prime}}$

where the summation is taken over all non-long, non-ordered compatible collections of 2-clusters $\left[S D_{1}, \ldots, S D_{m}\right], \bigcup_{i=1}^{m} \operatorname{supp}\left(S D_{i}\right) \subset V_{N}-V_{M}$ corresponding to the boundary conditions $\left\{\phi^{1}\left(\mathbf{Z}^{v}-V_{N}\right), \phi^{2}\left(\mathbf{Z}^{v}-V_{N}\right) ; \phi^{\prime}\left(V_{M}\right)\right.$ and $\left.\phi^{\prime \prime}\left(V_{M}\right)\right\}$.

In other words, in models with a not-long 2-clusters property the statistical weights of long 2-clusters are negligible.

We are ready to formulate our uniqueness criterion in its first form.

Theorem 1. Any model (1) having a not-long 2-clusters property has at most one limiting Gibbs state. 
We are going to reformulate the uniqueness criterion in a more applicable form (in terms of percolation).

Define a partition function $\Xi^{\phi^{1}, \phi^{\prime}, \phi^{2}, \phi^{\prime \prime},(n . l .)}$ as $\sum w\left(S D_{1}\right) \cdots w\left(S D_{m}\right)$, where the summation is taken over all non-ordered compatible collections of 2-clusters $\left[S D_{1}, \ldots, S D_{m}\right]$ containing at least one long 2-cluster, $\bigcup_{i=1}^{m}$ supp $D_{i} \subset V_{N}-V_{M}$ corresponding to the boundary conditions $\phi^{1}\left(\mathbf{Z}^{v}-V_{N}\right), \phi^{2}\left(\mathbf{Z}^{v}-V_{N}\right) ; \phi^{\prime}\left(V_{M}\right)$ and $\phi^{\prime \prime}\left(V_{M}\right)$.

Let us also define a partition function $\Xi^{\phi^{1}, \phi^{\prime}, \phi^{2}, \phi^{\prime \prime},(l .)}$ as $\sum w\left(S D_{1}\right) \cdots w\left(S D_{m}\right)$ where the

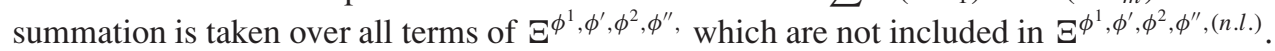

By dividing of both sides of the equality

$$
\Xi^{\phi^{1}, \phi^{\prime}, \phi^{2}, \phi^{\prime \prime}}=\Xi^{\phi^{1}, \phi^{\prime}, \phi^{2}, \phi^{\prime \prime},(n . l .)}+\Xi^{\phi^{1}, \phi^{\prime}, \phi^{2}, \phi^{\prime \prime},(l)}
$$

by $\Xi^{\phi^{1}, \phi^{\prime}, \phi^{2}, \phi^{\prime \prime}}$, we obtain

$$
1=\frac{\Xi^{\phi^{1}, \phi^{\prime}, \phi^{2}, \phi^{\prime \prime},(n . l .)}}{\Xi^{\phi^{1}, \phi^{\prime}, \phi^{2}, \phi^{\prime \prime}}}+\frac{\Xi^{\phi^{1}, \phi^{\prime}, \phi^{2}, \phi^{\prime \prime},(l)}}{\Xi^{\phi^{1}, \phi^{\prime}, \phi^{2}, \phi^{\prime \prime}}} .
$$

From definitions (12) for any model having a not-long 2-clusters property the absolute value of the second term of the previous equality (which is not necessarily positive) is less than $\epsilon$.

Consider

$$
\frac{\Xi^{\phi^{1}, \phi^{\prime}, \phi^{2}, \phi^{\prime \prime},(l)}}{\Xi^{\phi^{1}, \phi^{\prime}, \phi^{2}, \phi^{\prime \prime}}}=\frac{\Xi^{\phi^{1}, \phi^{\prime}, \phi^{2}, \phi^{\prime \prime},(l)}}{\Xi^{\phi^{1}, \phi^{\prime}, \phi^{2}, \phi^{\prime \prime},(n . l .)}+\Xi^{\phi^{1}, \phi^{\prime}, \phi^{2}, \phi^{\prime \prime},(l .)}} .
$$

If we replace each term belonging to $\Xi^{\phi^{1}, \phi^{\prime}, \phi^{2}, \phi^{\prime \prime},(l .)}$ by its absolute value, then $\Xi^{\phi^{1}, \phi^{\prime}, \phi^{2}, \phi^{\prime \prime},(l .)}$ transfers into $\Xi^{\phi^{1}, \phi^{\prime}, \phi^{2}, \phi^{\prime \prime},(l, a b s .)}$.

Since the sign of $\Xi^{\phi^{1}, \phi^{\prime}, \phi^{2}, \phi^{\prime \prime},(l .)}$ is not definite, we have (under the assumption that $\Xi^{\phi^{1}, \phi^{\prime}, \phi^{2}, \phi^{\prime \prime},(n . l .)}>\Xi^{\phi^{1}, \phi^{\prime}, \phi^{2}, \phi^{\prime \prime},(l ., a b s .)}$, which will follow from (13)):

$$
\begin{gathered}
-\frac{\Xi^{\phi^{1}, \phi^{\prime}, \phi^{2}, \phi^{\prime \prime},(l ., a b s .)}}{\left(\Xi^{\phi^{1}, \phi^{\prime}, \phi^{2}, \phi^{\prime \prime},(n . l .)}-\Xi^{\phi^{1}, \phi^{\prime}, \phi^{2}, \phi^{\prime \prime},(l ., a b s .)}\right)} \leqslant \frac{\Xi^{\phi^{1}, \phi^{\prime}, \phi^{2}, \phi^{\prime \prime},(l .)}}{\left(\Xi^{\phi^{1}, \phi^{\prime}, \phi^{2}, \phi^{\prime \prime},(n . l .)}+\Xi^{\phi^{1}, \phi^{\prime}, \phi^{2}, \phi^{\prime \prime},(l .)}\right)} \\
\leqslant \frac{\Xi^{\phi^{1}, \phi^{\prime}, \phi^{2}, \phi^{\prime \prime},(l ., a b s .)}}{\left(\Xi^{\phi^{1}, \phi^{\prime}, \phi^{2}, \phi^{\prime \prime},(n . l .)}+\Xi^{\phi^{1}, \phi^{\prime}, \phi^{2}, \phi^{\prime \prime},(l ., a b s .)}\right)} .
\end{gathered}
$$

Simple calculations show that inequality (12) follows from the following inequality:

$$
\frac{\Xi^{\phi^{1}, \phi^{\prime}, \phi^{2}, \phi^{\prime \prime},(l ., a b s .)}}{\left(\Xi^{\phi^{1}, \phi^{\prime}, \phi^{2}, \phi^{\prime \prime},(n . l .)}+\Xi^{\phi^{1}, \phi^{\prime}, \phi^{2}, \phi^{\prime \prime},(l ., a b s .)}\right)}<\epsilon / 2 .
$$

In the following the expression $\Xi^{\phi^{1}, \phi^{\prime}, \phi^{2}, \phi^{\prime \prime},(n . l .)}+\Xi^{\phi^{1}, \phi^{\prime}, \phi^{2}, \phi^{\prime \prime},(l ., a b s .)}$ will be denoted by $\Xi^{\phi^{1}, \phi^{\prime}, \phi^{2}, \phi^{\prime \prime}(a b s)}$. The expression $\Xi^{\phi^{1}, \phi^{\prime}, \phi^{2}, \phi^{\prime \prime},(l . a b s)} / \Xi^{\phi^{1}, \phi^{\prime}, \phi^{2}, \phi^{\prime \prime}(a b s)}$ can be interpreted as an 'absolute probability' $P^{a b s}$ (Long) of the event that there is at least one long 2-cluster.

Definition 2. We say that in model (1) 2-cluster percolation does not take place if a number $\epsilon, 0<\epsilon<1$ exists such that for each fixed cube $V_{M}$, a number $N_{0}=N_{0}(M)$, which depends on $M$ only exists such that if $N>N_{0}$ then (13) is held.

Note that from the above definitions any model in which 2-cluster percolation does not take place has a not-long super-clusters property.

Remark. In the spirit of the Kolmogorov '0-1 law' it can easily be shown that for any model in which super-cluster percolation does not take place for any $V_{M}$

$$
\lim _{N \rightarrow \infty} \frac{\Xi^{\phi^{1}, \phi^{\prime}, \phi^{2}, \phi^{\prime \prime},(l ., a b s .)}}{\left(\Xi^{\phi^{1}, \phi^{\prime}, \phi^{2}, \phi^{\prime \prime},(n . l .)}+\Xi^{\phi^{1}, \phi^{\prime}, \phi^{2}, \phi^{\prime \prime},(l ., a b s .)}\right)}=0 .
$$


Thus, for all models in which 2-cluster percolation does not take place, the probability that starting at any cube $V_{M}$ we can reach the boundary at infinity by all possible combinations of 2-clusters is zero.

Now we can formulate the main criterion for the absence of phase transitions.

Theorem 2. Any model (1) in which 2-cluster percolation does not take place has at most one limiting Gibbs state.

\section{Proof of the results}

In this section we prove theorem 1 , theorem 2 is a consequence of theorem 1 since any model in which super-cluster percolation does not take place has a not-long 2-clusters property.

Let $\phi_{N}^{\mathrm{min}, 1} \in \Phi(N)$ be a configuration with the minimal energy. The following lemma describes the structure of the configuration $\phi_{N}^{\min , 1}$.

Lemma 1. For arbitrary fixed boundary conditions $\phi^{1}$ a positive constant $N_{b}$ exists that does not depend on the boundary conditions $\phi^{1}$ and $N$, such that the restriction of the configuration $\phi_{N}^{\min , 1}$ to the cube $V_{N-N_{b}}$ coincides with the ground state $\phi^{g r}$.

Proof. Obviously, for each value of $N$ there is a number $N_{b}=N_{b}\left(N, \phi^{1}\right)\left(0 \leqslant N_{b} \leqslant N\right)$ satisfying the lemma, thus, the restriction of the configuration $\phi_{N}^{\min , 1}$ to the set $V_{N-N_{b}}$ coincides with the ground state $\phi^{g r}$.

Let $N_{b}\left(\left(N, \phi^{1}\right)\right.$ be minimal. Define $N_{b}(N)=\max _{\phi^{1}} N_{b}\left(N, \phi^{1}\right)$ where the maximum is taken over all possible boundary conditions $\phi^{1}$.

In order to prove the lemma we show that $\max _{N} N_{b}(N)$ is bounded.

Indeed, suppose that $\max _{N} N_{b}(N)$ is not bounded. Then a sequence of numbers $N(k)$, a sequence of boundary conditions $\phi^{k}(x) ; x \in \mathbf{Z}^{v}-V_{N(k)}$ and a corresponding sequence of configurations with minimal energy $\phi_{N(k)}^{\min , k}(x), k=1,2, \ldots$, exist such that $\lim _{k \rightarrow \infty} N(k)=\infty$ and $\lim _{k \rightarrow \infty} N_{b}\left(N(k), \phi^{k}\right)=\infty$.

For each $N(k)$ and $\phi^{k}$ define a point $z \in \mathbf{Z}^{v}$ maximally distanced from the boundary such that $\phi_{N(k)}^{\min , k}(x) \neq \phi^{g r}$.

Let us define a configuration $\psi_{N\left(k^{\prime}\right)}(x)=\phi_{N(k)}^{\min , k}(x-z)$.

Note that the restriction of the configurations $\psi_{N\left(k^{\prime}\right)}$ to any cube $V_{N}$ does not coincide with the ground state.

We say that a sequence of configurations $\psi_{V(k)}(x)$ converges pointwisely to the configuration $\psi(x)$, if for each $x \in \mathbf{Z}^{v}, k_{1}$ exists such that $\psi_{N(k)}(x)=\psi(x)$, if $k>k_{1}$.

After this natural definition, using a diagonal argument we can show that the sequence $\psi_{N\left(k^{\prime}\right)}(x), k^{\prime}=1,2, \ldots$ has at least one limit point, say $\psi^{\min }(x) \neq \phi^{g r}$. Indeed, suppose that $x_{1}, x_{2}, x_{3}, \ldots$ is the ordering of all points of $\mathbf{Z}^{v}$. Then a subsequence $\psi_{N\left(k^{\prime}\right)}^{x_{1}}$ of $\psi_{N\left(k^{\prime}\right)}$ exists, such that $\psi_{N\left(k^{\prime}\right)}^{x_{1}}\left(x_{1}\right)$ is a constant; a subsequence $\psi_{N\left(k^{\prime}\right)}^{x_{1}, x_{2}}$ of $\psi_{N\left(k^{\prime}\right)}^{x_{1}}$ exists such that $\psi_{N\left(k^{\prime}\right)}^{x_{1}, x_{2}}\left(x_{2}\right)$ is a constant; a subsequence $\psi_{N\left(k^{\prime}\right)}^{x_{1}, x_{2}, x_{2}}(x)$ of $\psi_{N\left(k^{\prime}\right)}^{x_{1}, x_{2}}$ exists such that $\psi_{N\left(k^{\prime}\right)}^{x_{1}, x_{3}}\left(x_{3}\right)$ is a constant; by continuing this process we obtain a subsequence $\psi_{N\left(k^{\prime}\right)}^{x_{1}, x_{2}, \ldots,}(x)$ of $\psi_{N(k)}$ which converges to a configuration of $\psi^{\mathrm{min}}$.

Note that $\psi^{\mathrm{min}}$ is a ground state. In fact, suppose that $\bar{\psi}$ is an arbitrary perturbation of $\psi^{\min }$ on a finite set $W$.

$$
H(\bar{\psi})-H\left(\psi^{\min }\right) \geqslant H_{N}\left(\bar{\phi} \mid \phi^{k^{\prime}}\right)-H_{N}\left(\phi^{\min } \mid \phi^{k^{\prime}}\right)-\epsilon\left(W, N(k), \phi^{k^{\prime}}\right)
$$

where $\bar{\phi}$ is the same perturbation of $\phi^{\text {min }}$ on the set $W-z$, and for each fixed $W$ the term $\epsilon\left(W, N\left(k^{\prime}\right), \phi^{k^{\prime}}\right)$ tends to zero uniformly with respect to $\phi^{k^{\prime}}$ while $N\left(k^{\prime}\right)$ tends to infinity. 
However, by construction $H_{N}\left(\bar{\phi} \mid \phi^{k^{\prime}}\right)-H_{N}\left(\phi^{\min , k^{\prime}} \mid \phi^{k^{\prime}}\right) \geqslant 0$. Therefore, $H(\bar{\phi})-$ $H\left(\phi^{\mathrm{min}}\right) \geqslant 0$ and $\psi^{\min }$ is a ground state.

Note that the configuration $\psi^{\min }(x) \neq \phi^{g r}(x)$. In fact, since the configuration $\psi_{V\left(k^{\prime}\right)}(x)$, which is just a shift of $\varphi_{V\left(k^{\prime}\right)}^{\min , k^{\prime}}$, the ground state $\varphi^{g r}$ cannot coincide with $\psi_{N\left(k^{\prime}\right)}(x)$ on the cube $V_{N}$. And $\psi^{\min }$ is a limit of the configurations $\psi_{N\left(k^{\prime}\right)}(x)$. This contradicts the assumption that $\max _{N} N_{b}(N)$ is not bounded. Therefore, lemma 1 is proved.

Let $\mathbf{P}^{1}$ and $\mathbf{P}^{2}$ be two extreme limiting Gibbs states corresponding to the boundary conditions $\phi^{1}$ and $\phi^{2}[21,22]$, and $\mathbf{P}_{N}^{1}$ and $\mathbf{P}_{N}^{2}$ are Gibbs distributions on $\Phi(N)$, corresponding to the boundary conditions $\phi^{1}$ and $\phi^{2}$.

Theorem 3 (See $[21,22]) . \mathbf{P}^{1}$ and $\mathbf{P}^{2}$ are singular or coincide.

We prove the uniqueness of the limiting Gibbs states of model (1) by showing that $\mathbf{P}^{1}$ and $\mathbf{P}^{2}$ are not singular.

Lemma 2. Limiting Gibbs states $\mathbf{P}^{1}$ and $\mathbf{P}^{2}$ are absolutely continuous with respect to each other.

Proof. Let $V_{M}$ be an arbitrary cube and $\phi^{\prime}\left(V_{M}\right)$ be an arbitrary configuration.

In order to prove the lemma we show that two positive constants $s_{0}$ and $S_{0}$ do not depend on $V_{M}, \phi^{1}, \phi^{2}$ and $\phi^{\prime}\left(V_{M}\right)$ exist, such that

$$
s_{0} \leqslant \mathbf{P}^{1}\left(\phi^{\prime}\left(V_{M}\right)\right) / \mathbf{P}^{2}\left(\phi^{\prime}\left(V_{M}\right)\right) \leqslant S_{0} .
$$

Let $\mathbf{P}_{N}^{1}$ and $\mathbf{P}_{N}^{2}$ be Gibbs distributions on $\Phi(N)$ corresponding to the boundary conditions $\phi^{1}$, and $\phi^{2}$. Thus,

$$
\lim _{N \rightarrow \infty} \mathbf{P}_{N}^{1}=\mathbf{P}^{1} \quad \text { and } \quad \lim _{N \rightarrow \infty} \mathbf{P}_{N}^{2}=\mathbf{P}^{2}
$$

where by convergence we mean the weak convergence of probability measures.

To establish inequality (14) we prove that for each fixed cube $V_{M}$, a number $N_{0}(M)$, depending on $M$ only exists such that for $N>N_{0}$

$$
s_{0} \leqslant \mathbf{P}_{V}^{1}\left(\phi^{\prime}\left(V_{M}\right)\right) / \mathbf{P}_{V}^{2}\left(\phi^{\prime}\left(V_{M}\right)\right) \leqslant S_{0} .
$$

The probability $\mathbf{P}_{V}^{1}\left(\varphi^{\prime}\left(V_{M}\right)\right)$ is given by (3). We can express $\mathbf{P}_{V}^{2}\left(\varphi^{\prime}\left(V_{M}\right)\right)$ in just the same way.

In order to prove inequality (15) it is enough to establish inequalities (16) and (17):

$$
0.9<Y\left(\varphi(I), V, \varphi^{i}\right)<1.1 \quad i=1,2
$$

and

$$
1 / S \leqslant\left(\frac{\Xi^{\phi^{1}, \phi^{\prime \prime}}}{\Xi^{\phi^{1}, \phi^{\prime}}}\right) /\left(\frac{\Xi^{\phi^{2}, \phi^{\prime \prime}}}{\Xi^{\phi^{2}, \phi^{\prime}}}\right) \leqslant 1 / s
$$

for arbitrary $\varphi^{\prime \prime}\left(V_{M}\right)$, where $S=(1.1 / 0.9)^{2} S_{0}$ and $s=(0.9 / 1.1)^{2} s_{0}$.

Indeed, if inequalities (16) and (17) hold, then

$$
1 /(1 / s) \leqslant \mathbf{P}_{V}^{1}\left(\varphi^{\prime}\left(V_{M}\right)\right) / \mathbf{P}_{V}^{2}\left(\varphi^{\prime}\left(V_{M}\right)\right) \leqslant 1 /(1 / S)
$$

since the quotient of $\left(\sum_{i=1}^{n} a_{i}\right) /\left(\sum_{i=1}^{n} b_{i}\right)$ lies between $\min \left(a_{i} / b_{i}\right)$ and $\max \left(a_{i} / b_{i}\right)$.

We now start to prove inequalities (16) and (17).

Inequality (16) is a direct consequence of the condition that the potential is a decreasing function: for each fixed $M$ there exists $N_{0}$, such that if $N>N_{0}$, then $0.9<Y\left(\phi(I), N, \phi^{i}\right)<$ $1.1 ; i=1,2$. 


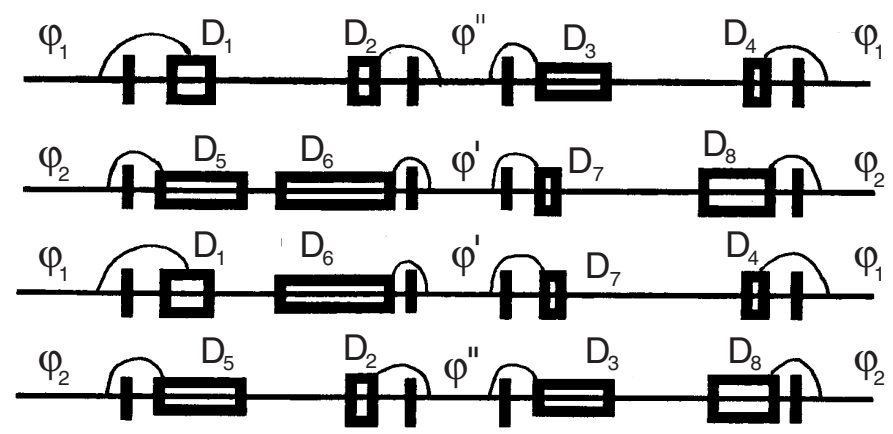

Figure 1. One-to-one correspondence between the terms of two double-partition functions.

So, in order to complete the proof of lemma 2 we have to establish the following inequality (which is just inequality (17) transformed):

$$
1 / S \leqslant \frac{\Xi^{\phi^{1}, \phi^{\prime \prime}} \Xi^{\phi^{2}, \phi^{\prime}}}{\Xi^{\phi^{2}, \phi^{\prime \prime}} \Xi^{\phi^{1}, \phi^{\prime}}} \leqslant 1 / s .
$$

We show that for each fixed cube $V_{M}$, a number $N_{0}(M)$, which depends on $M$ only exists such that if $N>N_{0}(M)$

$$
s \leqslant\left(\Xi^{\phi^{1}, \phi^{\prime}} \Xi^{\phi^{2}, \phi^{\prime \prime}}\right) /\left(\Xi^{\phi^{1}, \phi^{\prime \prime}} \Xi^{\phi^{2}, \phi^{\prime}}\right) \leqslant S
$$

for two positive constants $s$ and $S$ not depending on $M, \phi^{1}, \phi^{2}, \phi^{\prime}$ and $\phi^{\prime \prime}$.

Partition functions including only non-long super clusters satisfy the following key lemma which has a geometrically combinatorial explanation.

\section{Lemma 3 (See [18]).}

$$
\Xi^{\phi^{1}, \phi^{\prime \prime}, \phi^{2}, \phi^{\prime},(n . l .)}=Q \Xi^{\phi^{1}, \phi^{\prime}, \phi^{2}, \phi^{\prime \prime},(n . l .)}
$$

where the factor $Q=Q\left(\phi^{1}\left(\mathbf{Z}^{v}-V_{N}\right), \phi^{2}\left(\mathbf{Z}^{v}-V_{N}\right), \phi^{\prime}\left(V_{M}\right), \phi^{\prime \prime}\left(V_{M}\right)\right)$ is uniformly bounded:

$$
0<\text { constant }_{1}<Q<\text { constant }_{2} .
$$

Note that the factor, $Q$, appears because the configurations with minimal energies corresponding to the different boundary conditions do not coincide everywhere (due to lemma 1 they differ on a finite set and, due to condition 2, $Q$ is finite).

Proof. Due to the factor $Q$ without loss of generality we assume that the configurations with minimal energies corresponding to the different boundary conditions coincide with $\varphi^{g r}$.

The summations in $\Xi^{\phi^{1}, \phi^{\prime \prime}, \phi^{2}, \phi^{\prime},(n . l .)}=\Xi^{\phi^{1}, \phi^{\prime}, \phi^{2}, \phi^{\prime \prime},(n . l .)}$ are taken over all non-long, nonordered compatible collections of 2-clusters.

We put a one-to-one correspondence between the terms of these two double-partition functions.

Figure 1 shows how this one-to-one correspondence can be carried out.

We compare the term

$$
w\left(D_{1}^{1,,^{\prime \prime}}\right) w\left(D_{2}^{1,{ }^{\prime \prime}}\right) w\left(D_{3}^{1,{ }^{\prime \prime}}\right) w\left(D_{4}^{1,{ }^{\prime \prime}}\right) w\left(D_{5}^{2,{ }^{\prime}}\right) w\left(D_{6}^{2,{ }^{\prime}}\right) w\left(D_{7}^{2,{ }^{\prime}}\right) w\left(D_{8}^{2,{ }^{\prime}}\right)
$$

(the first four factors of this term came from the partition function $\Xi^{\phi^{1}, \phi^{\prime \prime}}$ and the last four factors of this term came from the partition function $\Xi^{\phi^{2}, \phi^{\prime \prime}}$ ) of the super-partition function $\Xi^{\phi^{1}, \phi^{\prime \prime}, \phi^{2}, \phi^{\prime},(n . l .)}$ to the term

$$
w\left(D_{1}^{1, \prime^{\prime}}\right) w\left(D_{6}^{1, \prime^{\prime}}\right) w\left(D_{7}^{1, \prime^{\prime}}\right) w\left(D_{4}^{1,{ }^{\prime}}\right) w\left(D _ { 5 } ^ { 2 , { } ^ { \prime \prime } } w ( D _ { 2 } ^ { 2 , , ^ { \prime \prime } } ) w \left(D_{3}^{2,,^{\prime \prime}} w\left(D_{8}^{2,,^{\prime \prime}}\right)\right.\right.
$$


(the first four factors of this term came from the partition function $\Xi^{\phi^{1}, \phi^{\prime}}$ and the last four factors of this term came from the partition function $\Xi^{\phi^{2}, \phi^{\prime \prime}}$ ) of the super-partition function $\Xi^{\phi^{1}, \phi^{\prime}, \phi^{2}, \phi^{\prime \prime},(n . l .)}$.

It can easily be shown that this one-to-one correspondence is well defined: if a term from $\Xi^{\phi^{1}, \phi^{\prime}, \phi^{2}, \phi^{\prime \prime},(n . l .)}$ corresponding to the term from $\Xi^{\phi^{1}, \phi^{\prime \prime}, \phi^{2}, \phi^{\prime},(n . l .)}$ does not exist (in other words, the corresponding clusters from $\Xi^{\phi^{1}, \phi^{\prime}}$ or $\Xi^{\phi^{2}, \phi^{\prime \prime}}$ are overlapped) then the term from $\Xi^{\phi^{1}, \phi^{\prime \prime}, \phi^{2}, \phi^{\prime},(n . l .)}$ is a long super cluster, which is impossible. Therefore, lemma 3 is proved. proved.

Inequality (19) is a direct consequence of (12) and lemma 3. Therefore, lemma 2 is

Proof of theorem 1. Let $\mathbf{P}^{1}$ and $\mathbf{P}^{2}$ be two different extreme limiting Gibbs states of model (1) corresponding to the boundary conditions $\phi^{1}$ and $\phi^{2}$ respectively. Due to lemma $2 \mathbf{P}^{1}$ and $\mathbf{P}^{2}$ are not singular. Thus, from theorem $3 \mathbf{P}^{1}$ and $\mathbf{P}^{2}$ coincide, which contradicts the assumption. Therefore, theorem 1 is proved.

The proof of the uniqueness criterion is based on two important points. The first point is the introduction of a contour model common for all boundary conditions. After that, by using a well known trick [20] we obtain 'non-interacting' clusters from interacting contours. The second point is the combinatorial lemma 3, which allows us to reduce the dependence of the expression $\mathbf{P}_{N}^{1}\left(\phi\left(V_{M}\right)\right) / \mathbf{P}_{N}^{2}\left(\phi\left(V_{M}\right)\right)$ on the boundary conditions $\phi^{1}$ and $\phi^{2}$ to the sum of the statistical weights of 2-clusters connecting the cube $V_{M}$ with the boundary (so-called long 2-clusters).

Theorems 1 and 2 have generalizations for non-translation-invariant potentials.

\section{Applications}

\subsection{One-dimensional models}

3.1.1. The problem of phase transitions in one-dimensional models with a long-range interaction has attracted the interest of many authors. In addition to the list of papers mentioned in the section 1, we would like to add [20-25]. It is well known that the condition $\sum_{r \in \mathbf{Z}^{1}, r>0} r|U(r)|<\infty(U(r)$ is a pair potential of long range) implies the uniqueness of limiting Gibbs states [14-17]. In the following we consider one-dimensional model under very natural regularity conditions and obtain the uniqueness result without this strong restriction on the potential of the model.

Condition 1. We say that the ground state $\phi^{g r}$ of model (1) satisfies the Peierls stability condition, if a constant $t$ exists such that for any finite set $A \subset \mathbf{Z}^{1} H\left(\phi^{\prime}\right)-H\left(\phi^{g r}\right) \geqslant t|A|$, where $|A|$ denotes the number of sites of $A$ and $\phi^{\prime}$ is a perturbation of $\phi^{g r}$ on the set $A$.

Condition 2. A constant $\gamma<1$ exists such that for any number $L$ and any interval $I=[a, b]$ with length $n$ and for any configuration $\phi(I)$

$$
\sum_{B \subset Z^{1} ; B \cap I \neq \emptyset, B \cap\left(Z^{1}-[a-L, b+L]\right) \neq \emptyset}|U(B)| \leqslant \text { constant } n^{\gamma} L^{\gamma-1} .
$$

This condition is very natural and, in particular, is held in models with pair potential $U(r) \sim 1 / r^{1+\delta}$, as $r \rightarrow \infty, \delta>0$.

Theorem 4. Suppose that $v=1$ and model (1) satisfies conditions 1 and 2. Then a value of the inverse temperature $\beta_{c r}$ exists such that if $\beta>\beta_{c r}$ then model (1) has at most one limiting Gibbs state. 
The conditions of theorem 4 are very natural. Phase transition takes place if some of these conditions are absent $[25,29]$.

Proof. In order to prove theorem 4 we show that for any model (1) $\beta_{c r}$ exists such that if $\beta>\beta_{c r}$ then in the model (1) 2-cluster percolation does not take place (theorem 3 ). In other words, we show that at low temperatures a number $\epsilon$ exists such that for each fixed $V_{M}$ (in our case interval $[-M, M]$ ) there exists a number $N_{0}$, which depends on $M$ only, such that if $N>N_{0}$ then the absolute probability (13) of long 2-clusters is less than $\epsilon$.

Long 2-clusters can connect the interval $\phi^{\prime}\left(V_{M}\right)$ or $\phi^{\prime \prime}\left(V_{M}\right)$ with $\phi^{1}$ or $\phi^{2}$.

It can easily be shown that in order to prove the theorem, it is sufficient to show that the probability that there is at least one 2 -cluster connecting $\phi(-\infty,-N)$ and $\phi^{\prime}[-M, M]$ is less than $\epsilon_{1}$, for $\epsilon_{1}<0$ at $\beta>\beta_{c r}$.

From the above definitions, the support of any 2-cluster is the union (connected by interaction elements) of contours or a heap of intersected contours, some sitting on others. In the following we call these contours and heaps of contours '2-contours' and denote them by $S K$.

We prove a stronger result asserting that the absolute probability of the event that there is a 2-contour connected to $\phi(-\infty,-N)$ by interaction elements is less than $\epsilon_{2}$ for $\epsilon_{2}<0$ at $\beta>\beta_{\text {cr }}$.

For each 2-contour $S K$ we define the notion of essential support ess $\operatorname{supp} K$. We say that an interval $[k, k+1]$ belongs to the essential support of $S K$ if, for at least one contour, $K^{\prime}=\left(\operatorname{supp} K^{\prime}, \phi^{\prime}\left(\operatorname{supp} K^{\prime}\right)\right.$ belonging to $S K \phi^{\prime}[k, k+1] \neq \phi^{g r}[k, k+1]$. We denote the number of unit $[k, k+1]$ intervals belonging to ess supp $S K$ by $|\operatorname{ess} \operatorname{supp} S K|$.

Suppose that the support of 2-cluster $S D$ consists of only 2-contour $S K$ (without any interaction elements). Then the statistical weight $w(S K)$ of this 2-cluster $S K$ is equal to $w(S K)=\exp (-\beta s|\operatorname{ess} \operatorname{supp} S K|)$ and by the straightforward application of Peierls argument it can be easily shown that the absolute probability of this 2-cluster

$$
P^{a b s}(S D)<\exp (-\beta s|\operatorname{ess} \operatorname{supp} S K|)
$$

where $s>0$ is a constant (actually $s=1-(1-t)(1-t)$ where $t$ is the Peierls constant, defined in condition 1).

We are going to estimate the absolute probability of the event that there is at least one 2-cluster connecting $\phi(-\infty,-N)$ and $\phi^{\prime}[-M, M]$. Suppose that the 2-cluster $S D$ is connected to $\phi(-\infty,-N)$. Let $S K$ be the leftmost 2-contour belonging to $S D$. We say that a 2-contour $S K^{\prime}$ is a neighbour of the first order of $S K$ and write $S K \leftrightarrow S K^{\prime}$ if $S K$ and $S K^{\prime}$ are connected by an interaction element. A 2-contour $S K^{\prime \prime}$ is called a neighbour of $q$ th order of $S K$ provided $S K \leftrightarrow S K_{1} \leftrightarrow S K_{2} \leftrightarrow \cdots \leftrightarrow S K_{q-1} \leftrightarrow S K^{\prime \prime}$ and there is no such diagram with fewer arrows. We are going to estimate $P^{a b s}(S D)$ by using the following method: in the first step we fix all 2-contours of order $q-1$ and take the summation over all 2-contours of order $q$, in the second step we fix all 2-contours of order $q-2$ and take the summation over all 2 -contours of order $q-1$, and so on. We repeat this summation $q-1$ times.

Proposition. Let $S K_{0}$ be a 2-contour of order $k$ and assume that for all 2-contours of order $k+1 w(S K)<\exp \left(-\frac{1}{2} \beta s|\operatorname{ess} \operatorname{supp} S K|\right)$. Then

$$
\sum_{S D: S D=\left(S K_{0}, S K, I G^{\prime}, I G^{\prime \prime}\right)} w(S D)<\exp \left(-\frac{1}{2} \beta s\left|\operatorname{ess} \operatorname{supp} S K_{0}\right|\right)
$$

at sufficiently large values of $\beta$. 
The above proposition states that if we fix a 2-contour and take the summation over all its neighbours then the constant $s$ in the statistical weight of this 2-cluster worsens at most to $s / 2$. The proof of the proposition is standard and is based on the technique of the restriction of entropy terms at low temperatures. We omit details (for a detailed proof in a special case see lemma 16 of [18]).

Now we are ready to estimate the absolute probability of the event that there is a 2contour $S K_{0}$ connected to $\phi(-\infty,-N)$. If we fix a 2-contour $S K_{0}$ and consider the set of all 2-clusters containing $S K_{0}$ as its leftmost 2-contour, then by applying the proposition we obtain the estimate:

$$
w\left(S K_{0}\right) \leqslant \exp \left(-\frac{1}{2} \beta s\left|\operatorname{ess} \operatorname{supp} S K_{0}\right|\right) .
$$

Suppose that $\mid$ ess supp $S K_{0} \mid=n$. From condition 2 the absolute probability of the event that $S K_{0}$ is connected to $\phi(-\infty,-N)$ is less than

$$
\sum_{L=0}^{\infty} \sum_{n=1}^{\infty} \exp \left(-\frac{1}{2} \beta s n\right)\left(\exp \left(\beta \text { constant } n^{\gamma} L^{\gamma-1}\right)-1\right)
$$

which in turn is less than any given $\epsilon$ at sufficiently large values of $\beta$.

Finally, since the absolute probability of percolation is less than the absolute probability of the event that $S K_{0}$ is connected to $\phi(-\infty,-N)$, the 2-cluster percolation does not take place. Therefore, theorem 4 follows from theorem 2.

Now we give a concrete application of theorem 2 .

Example. Consider a one-dimensional antiferromagnetical model with the Hamiltonian:

$$
H(\phi)=\sum_{x, y \in b f Z^{1}}|x-y|^{-1-\alpha} \phi(x) \phi(y)+h \sum_{x \in \mathbf{Z}^{1}} \phi(x)
$$

where the spin variables $\phi(x)$ take 0 and $1,0<\alpha<1$ and $h>0$.

One can easily show that the constant configuration $\phi=0$ is a unique ground state of model (21) and the model satisfies conditions 1 and 2 above. Thus, we can apply theorem 4 (or theorem 2) and prove that model (21) has unique limiting Gibbs states at low temperatures.

Note that due to the fact that model (21) has a very-long-range interaction, the result obtained is non-trivial and is not a consequence of classical methods and results: since

$$
\sum_{r \in \mathbf{Z}^{1}, r>0} r|U(r)|=\sum_{r \in \mathbf{Z}^{1}, r>0} r r^{-1-\alpha}=\infty
$$

the methods of $[14,15]$ are not applicable.

3.1.2. Based on theorem 2 we can prove uniqueness theorems at all temperature values if decreasing conditions on the interaction are stronger. But these cases are already covered in [14-17].

3.1.3. It would be very interesting to investigate the percolation by 2-clusters problem (consequently limiting the Gibbs state uniqueness problem) under natural conditions 1 and 2 at all temperature values. This problem currently seems to be difficult and will probably depend on obtaining more specific parameters of model (1).

\subsection{Two- and higher-dimensional models}

The analogue of theorem 4 can be proved for two-dimensional models. In two-dimensional models the standard cluster expansion method allows one to obtain the same result under the 
restriction that the potential has a short interaction range $(U(B)=0$ when $|B|$ is greater than a particular constant). Note that the value of $\beta_{c r}$ for two-dimensional case must be greater than the value of the critical inverse temperature for site percolation.

Theorem 5. Suppose that in model (1) site percolation does not take place and the value of $|g(G)|$ is uniformly less than 1. Then model (1) has at most one limiting Gibbs state.

The proof can be carried out using theorem 2. Actually, since there is no site percolation, for any long super cluster the number of interaction bonds $G$ uniformly tends to infinity when the volume $V_{N}$ increases. The absence of percolation by super clusters follows from the fact that $|g(G)|$ is uniformly less than 1 .

\section{Acknowledgments}

The author would like to thank the referees for their useful suggestions.

\section{References}

[1] Peierls R 1936 Proc. Camb. Phil. Soc. 32 477-8

[2] Bogolyubov N N and Khatset B I 1949 Dokl. Acad. Sci. 66 321-4

[3] Minlos R A and Sinai Ya G 1967 Trans. Mosc. Math. Soc. 17 237-68

[4] Minlos R A and Sinai Ya G 1968 Trans. Mosc. Math. Soc. 19 121-96

[5] Ruelle D 1969 Statistical Mechanics (New York: Benjamin)

[6] Gruber C and Kunz H 1971 Commun. Math. Phys. 22 133-61

[7] Fortuin C M and Kasteleyen P W 1972 Physica 57 536-64

[8] Pirogov S A and Sinai Ya G 1975 Teor. Math. Phys. 25 358-69

[9] Pirogov S A and Sinai Ya G 1975 Teor. Math. Phys. 26 61-76

[10] Glimm J, Jaffe A and Spencer T 1976 Ann. Phys., NY 101 610-30

[11] Glimm J, Jaffe A and Spencer T 1976 Ann. Phys., NY $101631-69$

[12] Malyshev V A 1980 Russian Math. Surveys 35 1-62

[13] Zahradnik M 1984 Commun. Math. Phys. 93 559-81

[14] Dobrushin R L 1968 Theor. Prob. Appl. 13 197-224

[15] Ruelle D 1968 Commun. Math. Phys. 9 267-78

[16] Dobrushin R L 1968 Funct. Anal. Appl. 2 302-12

[17] Dobrushin R L 1969 Funct. Anal. Appl. 3 22-8

[18] Kerimov A A 1993 J. Stat. Phys. 72 571-620

[19] Burkov S E and Sinai Ya G 1983 Russian Math. Surveys 38 235-57

[20] Bricmont J, Kuroda K and Lebowitz J L 1985 Commun. Math. Phys. 101 501-38

[21] Sinai Ya G 1982 Theory of Phase Transitions: Rigorous Results (Oxford: Pergamon)

[22] Georgii H-O 1988 Gibbs Measures and Phase Transitions (Berlin: de Gruyter)

[23] Dobrushin R L 1968 Teor. Veroyatn. Primen. 18 201-29

[24] Dobrushin R L 1968 Funk. Anal. Pril. 2 44-57

[25] Dyson F J 1969 Commun. Math. Phys. 12 91-107

[26] Frohlich J and Spencer T 1982 Commun. Math. Phys. 84 87-101

[27] Aizenman M, Chayes J T, Chayes L and Newman C M 1988 J. Stat. Phys. 50 1-40

[28] Berbee H 1989 Ann. Prob. 17 1416-31

[29] Kerimov A A and Sahin M A 1999 Physica A 265 169-75 